TRANSACTIONS OF THE

AMERICAN MATHEMATICAL SOCIETY

Volume 354, Number 12, Pages 4769-4788

S 0002-9947(02)03086-6

Article electronically published on July 25, 2002

\title{
SETS OF UNIQUENESS FOR SPHERICALLY CONVERGENT MULTIPLE TRIGONOMETRIC SERIES
}

\author{
J. MARSHALL ASH AND GANG WANG
}

\begin{abstract}
A subset $E$ of the $d$-dimensional torus $\mathbb{T}^{d}$ is called a set of uniqueness, or $U$-set, if every multiple trigonometric series spherically converging to 0 outside $E$ vanishes identically. We show that all countable sets are $U$-sets and also that $H^{J}$ sets are $U$-sets for every $J$. In particular, $C \times \mathbb{T}^{d-1}$, where $C$ is the Cantor set, is an $H^{1}$ set and hence a $U$-set. We will say that $E$ is a $U_{A}$-set if every multiple trigonometric series spherically Abel summable to 0 outside $E$ and having certain growth restrictions on its coefficients vanishes identically. The above-mentioned results hold also for $U_{A}$ sets. In addition, every $U_{A}$-set has measure 0 , and a countable union of closed $U_{A}$-sets is a $U_{A}$-set.
\end{abstract}

\section{Introduction and Main Results}

A subset $E$ of the $d$-dimensional torus $\mathbb{T}^{d}$ is called a set of uniqueness, or $U$-set, if whenever a multiple trigonometric series,

$$
\sum a_{\xi} e^{i\langle\xi, x\rangle},
$$

spherically converges to 0 outside $E$, that is, whenever the series satisfies

$$
\lim _{R \rightarrow \infty} \sum_{|\xi| \leq R} a_{\xi} e^{i\langle\xi, x\rangle}=0 \text { for all } x \in \mathbb{T}^{d} \backslash E,
$$

then the series vanishes identically, namely,

$$
a_{\xi}=0 \text { for all } \xi \in \mathbb{Z}^{d} .
$$

There is a rich one-dimensional theory of sets of uniqueness, even though there is no simple classification of sets of uniqueness. See the book of Kechris and Louveau $\mathrm{KL}$ for a good overview of this subject. Much of the best early work is summarized in chapter IX of Zygmund's Trigonometric Series, [Z1].

Some of the results appearing in Trigonometric Series have been extended to dimension 2 by Victor Shapiro, [Sh], [Sh3]. Here is a typical uniqueness theorem that is implicit in the work of Shapiro.

Theorem 1 (Shapiro). If $d=2$ and $\sum a_{\xi} e^{i\langle\xi, x\rangle}=0$ spherically at every point of the complement of a countable set $E \subset \mathbb{T}^{2}$, and if the coefficients satisfy

$$
a_{\xi} \rightarrow 0
$$

Received by the editors July 15, 1999 and, in revised form, April 24, 2000

2000 Mathematics Subject Classification. Primary 05C38, 15A15; Secondary 05A15, 15A18.

Key words and phrases. Abel summation, Baire category, Fourier series, generalized Laplacian, Green's function, $H^{J}$ sets, multiple trigonometric series, set of uniqueness, spherical convergence, subharmonic function, uniqueness.

This paper is in final form and no version of it will be submitted for publication elsewhere. 
so that, in particular,

$$
\sum_{R-1 \leq|\xi| \leq R}\left|a_{\xi}\right|=o(R),
$$

then the series vanishes identically.

Shapiro's work was done in the period 1957-1972. Because of work of Cooke [C, Zygmund [Z], and Connes [Co] in the 1970s and recent work of Bourgain [B], AW], it is now possible to prove all of Shapiro's theorems in all dimensions without the necessity of making assumption (1.1), or any other a priori assumptions about the coefficients. In other words, the above theorem may now be strengthened to this.

Theorem 2. Every countable subset of $\mathbb{T}^{d}$ is a $U$-set.

There exists an uncountable $U$-set once Theorem 2 holds. In fact, this is immediate from the axiom of choice, which implies that there exists an uncountable set that does not contain any perfect subset. Such a set must be a $U$-set because the set of points where a trigonometric series does not converge to 0 is a Borel set, and so, if it does not contain a perfect set, must be countable; by Theorem 2, this implies that the series vanishes identically. See reference [AW2] for details.

The following theorem gives constructive examples of uncountable perfect $U$-sets. Its proof avoids using the axiom of choice.

Theorem 3. Every $H^{J}$ subset of $\mathbb{T}^{d}$ is a $U$-set.

The definition of an $H^{J}$ set will be given in Section 3 below. For example, if $C$ is the Cantor set produced by partitioning $\mathbb{T}^{1}$ into 3 subintervals of equal length and proceeding recursively, then $C \times \mathbb{T}^{d-1}$ is an example of an $H^{1}$ set. One of the motivations for proving this theorem is that $H^{J}$ sets form a tower of ever "bigger" sets as $J$ increases. In fact, when $d=1$, Pyatetski-Shapiro has proved that for each $J=2,3, \ldots$, there are sets $H^{J}$ that are not countable unions of sets $H^{J-1}$, [PS, [PS1].

Remark 1. The definition of an $H^{J}$ set used here is essentially the one given by Victor Shapiro. Robert Kaufman has pointed out to us that various extensions of Pyatetski-Shapiro's definition of $H^{J}$ on $\mathbb{T}^{1}$ can be made to $\mathbb{T}^{d}$. We have not pursued any other extensions, because the goal of this paper is simply to produce a constructive example of a perfect $U$-set. By using Shapiro's definition, we are able to achieve this by combining the ideas in his paper [Sh3] with the methods of our earlier paper [AW1.

There is a large body of one-dimensional results concerning sets of uniqueness. Nevertheless, we will restrict ourselves mainly to the issues raised in Theorems 2 and 3 above. A partial result about whether a countable union of closed $U$-sets is still a $U$-set will be given in Theorem 8 in the next section. We end the introduction with the following remark. It is a natural question to ask if there exists a $U$-set that has positive measure. This is impossible if $d=1$. To see this, let $E \subset \mathbb{T}^{1}$ be a set of positive Lebesgue measure that is a $U$-set. Find a closed set $F \subset E,|F|>0$. Then, by the principle of localization, the Fourier series of the characteristic function of $F$ converges to 0 at each point of $F^{c}$, the complement of $F$, and so also at each point of $E^{c}$, since $E^{c} \subset F^{c}$. Thus $E$ is not a $U$-set. However, if $d \geq 2$, the principle of localization does not hold; so we do not know if a set of positive measure can be a $U$-set. This leads to the following conjecture. 
Conjecture 1. If $d \geq 2$, then no set of positive measure can be a U-set.

In the next section, we will introduce a related concept called sets of Abel uniqueness and use it to prove our main theorems.

\section{Sets of Abel Uniqueness and the Proofs of the Main Theorems}

The main goal of this work is the establishment of Theorems 2 and 3 . We find that Shapiro's path of establishing a theory of uniqueness for spherical Abel summability is useful when studying sets of uniqueness here. Our approach is to first study sets of uniqueness for spherical Abel summability with an additional condition. Then use the results obtained there to help us prove Theorems [2] and 3 ]

Historically, the major tool for studying sets of uniqueness is formal multiplication. When $d=1$, formal multiplication by a smooth function preserves convergence. In higher dimensions, we only know that formal multiplication by a smooth function transforms convergence to Bochner-Riesz summability of a certain order. Since Bochner-Riesz summability of any order implies Abel summability, this is why we study spherical uniqueness questions under Abel summation and consider sets of Abel uniqueness.

To give a reasonable definition of a set of Abel uniqueness, we need to impose certain conditions on the trigonometric series. When $d=1$, considering the example of $\delta^{\prime}(x)=-\sum \xi \sin \xi x$, which is spherically Abel summable to 0 at every $x \in \mathbb{T}^{1}$, shows that condition $a_{\xi}=o(|\xi|)$ is necessary for the empty set to be a set of Abel uniqueness. But, a more restrictive condition than $a_{\xi}=o(|\xi|)$ is needed to avoid the empty set being the only set of Abel uniqueness. Consider $\delta(x)=\frac{1}{2}+\sum_{\xi=1}^{\infty} \cos \xi x$. Note that $\delta$ is Abel summable to 0 except at the origin. Thus, we need a growth rate condition such as

$$
a_{\xi}=o(1) \text { as }|\xi| \rightarrow \infty
$$

in order to have any hope of including singletons as sets of Abel uniqueness. This partially motivates the following definition.

Definition 1 (Set of Abel Uniqueness). A set $E \subset \mathbb{T}^{d}$ is called a set of Abel uniqueness, or a $U_{A}$-set, if every multiple trigonometric series with coefficients satisfying

$$
\sum_{|\xi| \sim R}\left|a_{\xi}\right|^{2}=o\left(R^{2}\right) \text { as } R \rightarrow \infty, \text { and } a_{\xi}=o(1) \text { as }|\xi| \rightarrow \infty
$$

and spherically Abel summable to 0 outside $E$ is necessarily identically 0 . (The symbol $|\xi| \sim R$ means that $R / 2 \leq|\xi|<R$.)

In order to understand the origin of the condition

$$
\sum_{|\xi| \sim R}\left|a_{\xi}\right|^{2}=o\left(R^{2}\right) \text { as } R \rightarrow \infty,
$$

which we shall call Bourgain's condition, we begin with the following CantorLebesgue type theorem under spherical convergence.

Theorem 4 (Connes). Let $\mathcal{O} \subset \mathbb{T}^{d}$ be a ball or a subset that has full measure and is of Baire second category relative to $\mathbb{T}^{d}$. If $A_{R}(x)=\sum_{|\xi|=R} a_{\xi} e^{i\langle\xi, x\rangle}$ and the 
sequence $\left\{A_{R}(x)\right\}$ tends to 0 as $R \rightarrow \infty$ at every point of $\mathcal{O}$, then

$$
\sum_{|\xi|=R}\left|a_{\xi}\right|^{2}=o(1) \text { as } R \rightarrow \infty
$$

Connes proved this theorem for dimension $d$ in $1976[\mathrm{Co}$. Cooke [C] and shortly thereafter Zygmund [Z] had completed the $d=2$ case five years before Connes' work. For the history of the $d=1$ case, variants of which go back to Cantor, see [AKR]. Now, when a multiple trigonometric series $\sum a_{\xi} e^{i\langle\xi, x\rangle}$ $=\sum_{k=0}^{\infty}\left\{\sum_{|\xi|^{2}=k} a_{\xi} e^{i\langle\xi, x\rangle}\right\}=\sum_{k=0}^{\infty}\left\{A_{\sqrt{k}}(x)\right\}$ is spherically convergent at a point $x$, the sequence $\left\{A_{\sqrt{k}}(x)\right\}$ tends to 0 as $k \rightarrow \infty$. This leads immediately to the following corollary, which gives the coefficients' growth rate condition for spherically convergent multiple trigonometric series.

Corollary 1. Let $\mathcal{O} \subset \mathbb{T}^{d}$ be a ball or a subset that has full measure and is of Baire second category relative to $\mathbb{T}^{d}$. If

$$
\lim _{R \rightarrow \infty} \sum_{|\xi| \leq R} a_{\xi} e^{i\langle\xi, x\rangle}
$$

exists (as a finite number) at each point of $\mathcal{O}$, then

$$
\sum_{|\xi|=R}\left|a_{\xi}\right|^{2}=o(1) \text { as } R \rightarrow \infty
$$

Thus, both Bourgain's condition (2.3), which is nothing more than an averaging of Connes' condition (2.4) over the $O\left(R^{2}\right)$ possible values of $|\xi|$ that lie between $R / 2$ and $R$, and condition (2.1) are properties of series that converge on a substantial part of $\mathbb{T}^{d}$. So any uniqueness theorems proved for spherically Abel summable series, subject either to side condition (2.2) or side condition (2.4) will become a theorem without side conditions when restricted to the case of actual spherical convergence.

We make the choice of assuming (2.2) rather than (2.4) because both of the components in (2.2) are natural assumptions in the sense that they are stable under formal multiplication by a $C^{\infty}$ function. In fact, Lemma 10 in Section 3 shows that Bourgain's condition (2.3) is stable, while Lemma 1 of Berkovitz [Be] shows that condition (2.1) is also. But such stability seems to fail for Connes' condition (2.4).

This lack of stability seems to also happen with Shapiro's condition (1.1), which is what restricted his uniqueness theorems to the case $d=2$. Notice that both Shapiro's condition (1.1) and Bourgain's condition (2.3) follow from condition (2.1) if $d \leq 2$; while if $d \geq 3$, there are no connections between the three conditions (1.1), (2.1), and (2.3).

Remark 2. In view of Corollary 1, we see that if $E$ is a set of Abel uniqueness, then $E$ is also a set of uniqueness provided that its complement either contains a ball or has full measure and is of Baire second category relative to $\mathbb{T}^{d}$.

As we mentioned above, the failure of localization when $d \geq 2$ means that we cannot immediately reject sets of positive measure from consideration as possible sets of uniqueness. However, the situation is much simpler when we turn to the same question in the Abel setting.

Theorem 5. If $E \subset \mathbb{T}^{d}$ is a $U_{A}$-set, then $E$ has measure 0 . 
Proof. Assume that $|E|>0$. Since every positive measure set has a closed subset of positive measure, we may assume that $E$ is closed when thought of as a subset of the torus, i.e., that $E^{*}=\bigcup_{\mu}[E+2 \pi \mu]$ is closed in $\mathbb{R}^{d}$. Note that the characteristic function of $E, \chi_{E}$, is in $L^{2}\left(\mathbb{T}^{d}\right)$. Thus, the coefficients $a_{\xi}$ of the Fourier series of $\chi_{E}$ satisfy condition (2.2) since

$$
\sum_{\xi}\left|a_{\xi}\right|^{2}=\left\|\chi_{E}\right\|^{2}=|E|<\infty .
$$

Because $E^{*}$ is closed, the Fourier series of $\chi_{E}$ is spherically Abel summable to 0 off $E$ by Theorem 2 of [Sh1]. Thus $a_{\xi}=0$ for all $\xi$ since $E$ is a $U_{A}$-set. Consequently, $|E|=0$, by equation (2.5), which is a contradiction.

It is already known that any singleton is a set of Abel uniqueness. In fact, the following theorem appears as Theorem 1.8 in AW1.

Theorem 6. Consider the multiple $(d \geq 2)$ trigonometric series $\sum_{\xi \in \mathbb{Z}^{d}} a_{\xi} e^{i\langle\xi, x\rangle}$ where the coefficients $a_{\xi}$ are arbitrary complex numbers. Let $q$ be a point on $\mathbb{T}^{d}$. Suppose that

1. $\sum_{|\xi| \sim R}\left|a_{\xi}\right|^{2}=o\left(R^{2}\right)$ as $R \rightarrow \infty$,

2. $f^{*}(x)$ and $f_{*}(x)$ are finite for all $x$ except possibly on set $A=\{q\}$, and

3. $f^{*}(x)$ and $f_{*}(x)$ are functions in $L^{1}\left(\mathbb{T}^{d}\right)$.

Then $\sum_{\xi \in \mathbb{Z}^{d}} a_{\xi} e^{i\langle\xi, x\rangle}$ is the Fourier series of $f^{*}(x)$.

Here $f^{*}(x)$ denotes $\limsup \mathcal{R} f(x, t)+i \limsup \mathcal{I} f(x, t)$, where

$$
\begin{aligned}
f(x, t) & =\mathcal{R} f(x, t)+\mathcal{I} f(x, t) \\
& =\sum_{\xi \in \mathbb{Z}^{d}} a_{\xi} e^{i\langle\xi, x\rangle-|\xi| t},
\end{aligned}
$$

and $f_{*}(x)$ is defined similarly. We do not specify the spherical nature of the convergence of the series defining $f(x, t)$ here since the assumed condition (2.3) implies that the series converges absolutely and hence unconditionally. Note first that if set $A=\emptyset$, then the theorem holds true. Note also that the theorem is false when $d=1$ since the trigonometric series $\frac{1}{2}+\sum_{\xi>0} \cos \xi x$ is Abel convergent to 0 everywhere in $\mathbb{T}^{1} \backslash\{0\}$.

Apply this theorem to the special case where $f^{*}(x)=f_{*}(x)=0$ for every $x \neq q$ to see that singletons are $U_{A}$-sets.

Corollary 2. Consider the multiple $(d \geq 2)$ trigonometric series $\sum_{\xi \in \mathbb{Z}^{d}} a_{\xi} e^{i\langle\xi, x\rangle}$ where the coefficients $a_{\xi}$ are arbitrary complex numbers. Let $q$ be a point on $\mathbb{T}^{d}$. Suppose that the series spherically converges everywhere except at $q$ to an everywhere finite function $f(x) \in L^{1}\left(\mathbb{T}^{d}\right)$. Then $\sum_{\xi \in \mathbb{Z}^{d}} a_{\xi} e^{i\langle\xi, x\rangle}$ is the Fourier series of $f(x)$.

This follows immediately from Theorem [6, Remark [2, and the fact that spherical convergence implies spherical Abel summability. Therefore, any singleton is also a set of uniqueness. By considering formal multiplication of trigonometric series, we will also show in Sections 4 and 5, respectively, the following two theorems.

Theorem 7. For any dimension $d$ and positive integer $J, H^{J}$ sets are $U_{A}$-sets. 
Theorem 8. Suppose that $\left\{E_{k}\right\}_{k \geq 1}$ is a sequence of $U_{A}$-sets, and for each $k, E_{k}$ is closed in the sense that $E_{k}^{*}=\bigcup_{\mu}\left[E_{k}+2 \pi \mu\right]$ is closed in $\mathbb{R}^{d}$. Then $E=\bigcup_{k \geq 1} E_{k}$ is a $U_{A}$-set.

In Section 3 we will show that, in general, the complement of an $H^{J}$-set is dense in $\mathbb{T}^{d}$, hence has full measure and is of the second category in the sense of Baire. Therefore, by Remark 2, we get Theorem 3 as a corollary of Theorem 7 , Theorem (7)and Theorem 3 answer a question raised by Shapiro more than twenty years ago [Sh3]. Combining Theorem [6] and Theorem [8, we have the following corollary for sets of Abel uniqueness:

Corollary 3. Any countable set is a $U_{A}$-set.

Now simply apply Remark 2 to prove Theorem 2 We end this section with the following remark.

Remark 3. An interesting alternative to our definition of $U_{A}$-set would be to require only Bourgain's condition (2.3) but not condition (2.1). Tracking our proofs of Theorem [5. Theorem 8, and Corollary 3 shows that they would still hold. However, Theorem 7 would not. In particular, the linear set $\{(0, y)\}$ is easily shown to be an $H^{1}$-set, but the example $S(x, y)=\delta(x)$ shows that this set would not be a $U_{A}$-set. So for the existence of uncountable $U_{A}$-sets we would have to rely upon the nonconstructive axiom of choice dependent argument given above after the statement of Theorem 2 ,

\section{Preliminaries}

Before we define $H^{J}$ sets, we summarize some basic properties of normal sequences.

Definition 2. For any $J \geq 1$, we say that $\left\{n_{k}\right\}_{k \geq 1}=\left\{\left(n_{k}^{1}, \cdots, n_{k}^{J}\right)\right\}_{k \geq 1}$ is a onedimensional normal sequence of degree of freedom $J$ if for every $h=\left(h_{1}, \cdots, h_{J}\right) \in$ $\mathbb{Z}^{J} \backslash\{0\}$, we have

$$
\lim _{k \rightarrow \infty}\left|h_{1} n_{k}^{1}+\cdots+h_{J} n_{k}^{J}\right|=\lim _{k \rightarrow \infty}\left|\left\langle h, n_{k}\right\rangle\right|=\infty
$$

We omit the degree of freedom of a normal sequence if it is apparent.

Lemma 1. Let $\sigma=\left\{n_{k}\right\}_{k \geq 1}=\left\{\left(n_{k}^{1}, \cdots, n_{k}^{J}\right)\right\}_{k \geq 1}$ be a one-dimensional normal sequence. There exists a subsequence $\sigma^{\prime}=\left\{n_{k}^{\prime}\right\}_{k=1}^{\infty}$ such that for every $h \in \mathbb{Z}^{J} \backslash$ $\{0\},\left\{\left\langle h, n_{k}^{\prime}\right\rangle\right\}_{k \geq 1}$ is a set of distinct integers, except for a finite subset which depends on $h$.

Proof. This is a straightforward diagonalization argument. Order $\mathbb{Z}^{J} \backslash\{0\}=$ $\left\{h_{1}, h_{2}, \cdots\right\}$. Let $n_{11}=n_{1}$. Having chosen $n_{11}, \cdots, n_{1, j-1}$, use (3.1) to find $n_{1 j}$ as the first $n_{k}$ such that $\left|\left\langle h_{1}, n_{k}\right\rangle\right| \notin\left\{\left|\left\langle h_{1}, n_{11}\right\rangle\right|, \cdots,\left|\left\langle h_{1}, n_{1, j-1}\right\rangle\right|\right\}$. This way, we get a subsequence $\left\{n_{1 j}\right\}$ of $\left\{n_{k}\right\}$ such that $\left\{\left\langle h_{1}, n_{1 j}\right\rangle\right\}$ are distinct integers for all $j \geq 1$.

Now start with $h_{2}$ in place of $h_{1}$ and $\left\{n_{1 j}\right\}$ in place of $\left\{n_{k}\right\}$ and select a subsequence $\left\{n_{2 j}\right\}$ of $\left\{n_{1 j}\right\}$ so that $\left\{\left\langle h_{2}, n_{2 j}\right\rangle\right\}$ are distinct integers for all $j \geq 1$. Continue inductively: having selected $\left\{n_{i-1, j}\right\}_{j \geq 1}$ so that $\left\{\left\langle h_{i-1}, n_{i-1, j}\right\rangle\right\}_{j \geq 1}$ are a set of distinct integers, select $\left\{n_{i j}\right\}_{j \geq 1}$, a subsequence of $\left\{n_{i-1, j}\right\}_{j \geq 1}$ so that $\left\{\left\langle h_{i}, n_{i, j}\right\rangle\right\}_{j \geq 1}$ are a set of distinct integers. Finally, consider the subsequence $\left\{n_{j}^{\prime}\right\}=\left\{n_{j j}\right\}$. 
Given any $h_{o} \in \mathbb{Z}^{J} \backslash\{0\}$, there is an integer $j_{o}$ so that $h_{o}=h_{j_{o}}$. Since every $n_{j}^{\prime}$ for $j \geq j_{o}$ was selected from $\left\{n_{j_{o} k}\right\}_{k \geq j_{o}}$, the elements of $\left\{\left\langle h_{o}, n_{j}^{\prime}\right\rangle\right\}_{j \geq j_{o}}$ are distinct integers. Hence at most $j_{o}-1$ of these can agree with some number in the finite set $A=\left\{\left\langle h_{o}, n_{j}^{\prime}\right\rangle\right\}_{j=1}^{j_{o}-1}$. Discard these and, if necessary, some of the elements of $A$. All of the remaining elements of $\left\{\left\langle h_{o}, n_{j}^{\prime}\right\rangle\right\}_{j \geq 1}$ are distinct.

Definition 3. For any $J \geq 1$, say that

$$
\left\{n_{1 k}, \cdots, n_{d k}\right\}_{k \geq 1}=\left\{\left(n_{1 k}^{1}, \cdots, n_{1 k}^{J}, n_{2 k}^{1}, \cdots, n_{2 k}^{J}, \cdots, n_{d k}^{1}, \cdots, n_{d k}^{J}\right)\right\}_{k \geq 1}
$$

is a $d$-dimensional normal sequence of degree of freedom $J$ if each of the $d$ sequences $\left\{n_{1 k}\right\}_{k \geq 1}, \cdots,\left\{n_{d k}\right\}_{k \geq 1}$ is a one-dimensional normal sequence of degree of freedom $J$.

Lemma 2. Let $\sigma=\left\{n_{1 k}, \cdots, n_{d k}\right\}_{k \geq 1}$ be a d-dimensional normal sequence of degree of freedom $J$. Then there is a subsequence $\sigma^{\prime}=\left\{n_{1 k}^{\prime}, \cdots, n_{d k}^{\prime}\right\}_{k \geq 1}$ of $\sigma$ so that for any $j \in \mathbb{Z}^{d J} \backslash\{0\}$ and any $c \in\{1,2, \cdots, d\}$ either

$$
j_{c}=\left(j_{(c-1) J+1}, \cdots, j_{(c-1) J+J}\right)=0
$$

or

$$
\left\{\left\langle j_{c}, n_{c k}^{\prime}\right\rangle\right\}_{k \geq 1}=\left\{j_{(c-1) J+1} n_{c k}^{\prime 1}+\cdots+j_{(c-1) J+J} n_{c k}^{\prime J}\right\}_{k \geq 1}
$$

is a set of distinct integers, except for a finite subset that depends on $j$.

Proof. Apply Lemma 1 to $\left\{n_{1 k}\right\}_{k \geq 1}$, producing $\left\{n_{1 k_{\ell}}\right\}_{\ell \geq 1}$, the dot product of which with any $j \in \mathbb{Z}^{J} \backslash\{0\}$ has all but a finite number of terms distinct. Now note that a subsequence of a normal sequence is normal, start with $\left\{n_{2 k_{\ell}}\right\}_{\ell \geq 1}$, and apply Lemma 1 again. Then repeat this process another $d-2$ times. The resultant subsequence,

$$
\left\{\left(n_{1 k_{\ell_{1} \ell_{d}}}, \cdots, n_{d \ell_{\ell_{\cdots} \ell_{d}}}\right)\right\}_{\ell_{d} \geq 1}
$$

has the desired property.

Lemma 3. Let

$$
\sigma=\left\{\sigma_{k}\right\}_{k \geq 1}=\left\{n_{1 k}, \cdots, n_{d k}\right\}_{k \geq 1}=\left\{\left(n_{1 k}^{1}, \cdots, n_{1 k}^{J}, \cdots, n_{d k}^{1}, \cdots, n_{d k}^{J}\right)\right\}_{k \geq 1}
$$

be a sequence of elements of $\mathbb{Z}^{J d}$ and for $x=\left(x_{1}, \cdots, x_{d}\right) \in \mathbb{T}^{d}$, let

$$
\begin{aligned}
\sigma \otimes x & =\left\{\sigma_{k} \otimes x\right\}_{k \geq 1} \\
& =\left\{\left(n_{1 k}^{1} x_{1}, \cdots, n_{1 k}^{J} x_{1}, n_{2 k}^{1} x_{2}, \cdots, n_{2 k}^{J} x_{2}, \cdots, n_{d k}^{1} x_{d}, \cdots, n_{d k}^{J} x_{d}\right)\right\}_{k \geq 1} \\
& =\left\{x_{1} n_{1 k}, x_{2} n_{2 k}, \cdots, x_{d} n_{d k}\right\}_{k \geq 1} .
\end{aligned}
$$

If $\sigma$ is a d-dimensional normal sequence of degree of freedom $J$, then for almost every $x \in \mathbb{T}^{d}, \sigma \otimes x$ is dense in $\mathbb{T}^{d J}$.

Proof. It suffices to show that there is a subsequence $\sigma^{\prime}$ of $\sigma$, so that for all $j \in$ $\mathbb{Z}^{d J} \backslash\{0\}$,

$$
\frac{1}{N} \sum_{k=1}^{N} e^{2 \pi i\left\langle j, \sigma_{k}^{\prime} \otimes x\right\rangle} \rightarrow 0
$$


for almost every $x \in \mathbb{T}^{d}$. In fact, let $E_{j}$ be the set where condition (3.2) holds. Then

$$
\left|\bigcap_{j \in \mathbb{Z}^{d J} \backslash\{0\}} E_{j}\right|=\left|\mathbb{T}^{d}\right| .
$$

By the multidimensional Weyl criterion ( $\mathrm{KN}$, page 48, Theorem 6.2), for every $x \in \bigcap E_{j}, \sigma^{\prime} \otimes x$ is uniformly distributed, hence $\sigma \otimes x$ is dense.

To prove (3.2), fix $j=\left(j_{1}, \cdots, j_{d}\right) \in \mathbb{Z}^{d J} \backslash\{0\}$, where $j_{i}, i=1, \cdots, d$ is a vector of length $J$. If $j_{i} \neq 0$ for all $1 \leq i \leq d$, let $c=d$. Otherwise, since $j \neq 0$, there exists $1 \leq c<d$ such that by rearranging $j_{1}, \cdots, j_{d}$ if necessary, we have $j_{i} \neq 0$ for $1 \leq i \leq c$ and $j_{i}=0$ for $i>c$. By Lemma 2 there is an integer $M$ depending on $j$ and a subsequence $\sigma^{\prime}=\left(n_{1 k}^{\prime}, \cdots, n_{d k}^{\prime}\right)$ of $\sigma$ independent of $j$ such that for each $1 \leq i \leq c,\left\{\left\langle j_{i}, n_{i k}^{\prime}\right\rangle\right\}_{k \geq M}$ is a set of distinct integers. Let $a_{i k}=\left\langle j_{i}, n_{i k}^{\prime}\right\rangle, 1 \leq i \leq c$. Then

$$
\begin{aligned}
\left\langle j, \sigma_{k}^{\prime} \otimes x\right\rangle & =x_{1}\left\langle j_{1}, n_{1 k}^{\prime}\right\rangle+x_{2}\left\langle j_{2}, n_{2 k}^{\prime}\right\rangle+\cdots+x_{c}\left\langle j_{c}, n_{c k}^{\prime}\right\rangle \\
& =a_{1 k} x_{1}+a_{2 k} x_{2}+\cdots+a_{c k} x_{c} .
\end{aligned}
$$

Thus, (3.2) follows if we can show that

$$
f_{N}\left(x_{1}, \cdots, x_{c}\right)=\frac{1}{N} \sum_{k=1}^{N} e^{2 \pi i\left(a_{1 k} x_{1}+\cdots+a_{c k} x_{c}\right)} \rightarrow 0
$$

for almost every $\left(x_{1}, \cdots, x_{c}, x_{c+1}, \cdots, x_{d}\right)$. Since $x_{c+1}, \cdots, x_{d}$ have no effect on whether condition (3.3) holds, it suffices to show that condition (3.3) holds for almost every $\left(x_{1}, \cdots, x_{c}\right) \in \mathbb{T}^{c}$. Since $a_{i k}=\left\langle j_{i}, n_{i k}^{\prime}\right\rangle$ are distinct when $1 \leq i \leq c$ and $k \geq M$, by orthogonality we get

$$
\begin{aligned}
\int_{\mathbb{T}^{c}}\left|f_{N}\right|^{2} d x \leq & \frac{1}{N^{2}} \sum_{j=M}^{N} \sum_{k=M}^{N} \int_{0}^{1} \cdots \int_{0}^{1} e^{2 \pi i\left(a_{1 j}-a_{1 k}\right) x_{1}} \cdots e^{2 \pi i\left(a_{c j}-a_{c k}\right) x_{c}} d x_{1} \cdots d x_{c} \\
& \quad+\frac{1}{N^{2}} \sum_{j=1}^{N} \sum_{k=1}^{M-1} 1+\frac{1}{N^{2}} \sum_{j=1}^{M-1} \sum_{k=M}^{N} 1 \\
= & \frac{1}{N^{2}}((N-M+1)+N(M-1)+(M-1)(N-M+1)) \\
\leq & \frac{2 M}{N}
\end{aligned}
$$

From this it follows that the function $\sum_{n \geq 1}\left|f_{n^{2}}\left(x_{1}, \cdots, x_{c}\right)\right|^{2}$ is integrable over $\mathbb{T}^{c}$, hence finite for almost every $x \in \mathbb{T}^{c}$, so that in particular,

$$
f_{n^{2}}\left(x_{1}, \cdots, x_{c}\right) \rightarrow 0
$$

for almost every $x \in \mathbb{T}^{c}$. Pick such an $x$. Given any integer $N$, find $n$ so that $n^{2}<N \leq(n+1)^{2}$ and make the estimates

$$
\begin{aligned}
\left|f_{N}(x)\right| & =\left|\frac{n^{2}}{N} \frac{1}{n^{2}} \sum_{k=1}^{n^{2}} e^{2 \pi i\left(a_{1 k} x_{1}+\cdots+a_{c k} x_{c}\right)}+\frac{1}{N} \sum_{k=n^{2}+1}^{N} e^{2 \pi i\left(a_{1 k} x_{1}+\cdots+a_{c k} x_{c}\right)}\right| \\
& \leq\left|f_{n^{2}}(x)\right|+\left|\frac{1}{N} \sum_{k=n^{2}+1}^{N} 1\right| \\
& \leq\left|f_{n^{2}}(x)\right|+O\left(N^{-1 / 2}\right)
\end{aligned}
$$


to see that condition (3.3) holds at $x$.

Definition 4. Let $J \geq 1$. We say that a set $E \subset \mathbb{T}^{d}$ is a set of type $H^{J}$ if there exists a $d$-dimensional normal sequence $\sigma=\left\{\sigma_{k}\right\}_{k \geq 1}$ of degree of freedom $J$ and a nonempty domain $\mathcal{D} \subset \mathbb{T}^{d J}$ such that for all $x \in E, \sigma_{k} \otimes x \notin \mathcal{D}$ for all $k \geq 1$.

For example, the Cantor set is an $H^{1}$ set in $d=1$ corresponding to $\sigma=\left\{3^{k}\right\}$ and $\mathcal{D}=\left(\frac{2 \pi}{3}, \frac{4 \pi}{3}\right)$. The following two lemmas summarize the properties of $H^{J}$ needed in our paper.

Lemma 4. The closure of an $H^{J}$ set is also an $H^{J}$ set, has measure 0, and is nowhere dense. Consequently, the complement of an $H^{J}$ set is of the second category in the sense of Baire and has full measure.

Proof. Let $\bar{E}$ be the closure of an $H^{J}$ set $E$. From the definition and the fact that the function $f_{k}(x)=\sigma_{k} \otimes x \bmod 2 \pi$ is continuous at $x$ where $f_{k}(x) \in \mathcal{D}$, we have that $\bar{E}$ is also an $H^{J}$ set. By Lemma 3, it is obvious that $|\bar{E}|=0$. Also, $\bar{E}$, like any closed measure 0 set, is nowhere dense and hence of the first category. Thus the complement of $\bar{E}$, and consequently the complement of $E$, is of the second category.

The next lemma can be proved by an argument given by Shapiro (pp. 140-142 of [Sh3]).

Lemma 5. Let $H$ be an $H^{J}$ set. There exists a sequence of $C^{\infty}\left(\mathbb{R}^{d}\right)$ functions $\left\{\lambda_{k}(x)\right\}_{k \geq 1}$, of period $2 \pi$ in each variable, having the following properties:

1. for each $k \geq 1$, there exists a set $D_{k}^{*}=\bigcup_{\mu}\left[D_{k}+2 \pi \mu\right]$ open in $\mathbb{R}^{d}$ such that $H^{*}=\bigcup_{\mu}[H+2 \pi \mu] \subset D_{k}^{*}$ and $\lambda_{k}$ vanishes on $D_{k}^{*}$;

2. for each $k$, the Fourier coefficients of $\lambda_{k},\left\{\alpha_{\xi}^{k}\right\}_{\xi}$, satisfy $\alpha_{\xi}^{k}=o\left(|\xi|^{-(8 d+4)}\right)$ as $|\xi| \rightarrow \infty$

3. there exists a finite constant $C$ such that $\sum_{\xi}\left|\alpha_{\xi}^{k}\right| \leq C$ for all $k \geq 1$;

4. $\lim _{k \rightarrow \infty} \alpha_{\xi}^{k}=0$ for $\xi \neq 0$;

5. $\lim _{k \rightarrow \infty} \alpha_{0}^{k}=1$.

We say that a multiple series $\sum_{\xi} a_{\xi}$ is Bochner-Riesz summable of order $\gamma \geq 0$, denoted by summable $(B-R, \gamma)$, to $A$ if

$$
\lim _{R \rightarrow \infty} \sum_{|\xi| \leq R} a_{\xi}\left(1-|\xi|^{2} / R^{2}\right)^{\gamma}=A .
$$

It is easy to see that if $\sum_{\xi} a_{\xi}$ is summable $(B-R, \gamma)$ to $A$, then $\sum_{\xi} a_{\xi}$ is summable $(B-R, \gamma+\varepsilon)$ to $A$ for any $\varepsilon \geq 0$, and $\sum_{\xi} a_{\xi}$ is also spherically Abel summable to A.

For two multiple trigonometric series $S_{k}(x)=\sum_{\xi} a_{\xi}^{k} e^{i\langle\xi, x\rangle}, k=1,2$, the formal multiplication of $S_{1}(x)$ and $S_{2}(x), S_{3}(x)=S_{1}(x) S_{2}(x)$ is a multiple trigonometric series given by $S_{3}(x)=\sum_{\xi} a_{\xi}^{3} e^{i\langle\xi, x\rangle}$, where $a_{\xi}^{3}=\sum_{\pi} a_{\pi}^{1} a_{\xi-\pi}^{2}$.

The following result given by Berkovitz (Theorem $2^{\prime}$, Theorem 4, and Theorem 5 of $[\mathrm{Be}]$ ) on formal multiplication of multiple trigonometric series is well known.

Lemma 6. a) Let $S(x)$ be a multiple trigonometric series with coefficients $a_{\xi}=$ $o\left(|\xi|^{\gamma}\right), \gamma \geq-(d-1)$. Let $S^{\prime}(x)$ be a $C^{\infty}\left(\mathbb{T}^{d}\right)$ function of period $2 \pi$ for each variable and $\left\{a_{\xi}^{\prime}\right\}_{\xi}$ be its Fourier coefficients. If $S^{\prime}(x)$ has the constant value $C$ on $\mathcal{D}$, where 
$\mathcal{D}$ is a domain in $\mathbb{T}^{d}$, then $T(x)-C S(x)$ is summable $(B-R, \gamma+d-1)$ to 0 uniformly in $\mathcal{D}$, where $T(x)$ is the formal multiplication of $S(x)$ with $S^{\prime}(x)$.

b) Let $S(x)=\sum_{\xi} a_{\xi} e^{i\langle\xi, x\rangle}$ and $S^{\prime}(x)=\sum_{\xi} a_{\xi}^{\prime} e^{i\langle\xi, x\rangle}$ be d-dimensional multiple trigonometric series with coefficients $o\left(|\xi|^{\gamma}\right), \gamma>-(d-1)$. For $k=\lceil\gamma / 2\rceil+\lceil d / 2\rceil+$ 1, suppose

$$
F(x)=a_{0}|x|^{2 k} / c_{k}+\sum_{|\xi| \neq 0} \frac{(-1)^{k} a_{\xi}}{|\xi|^{2 k}} e^{i\langle\xi, x\rangle}
$$

and

$$
F^{\prime}(x)=a_{0}^{\prime 2 k}|x|^{2 k} / c_{k}+\sum_{|\xi| \neq 0} \frac{(-1)^{k} a_{\xi}^{\prime}}{|\xi|^{2 k}} e^{i\langle\xi, x\rangle},
$$

where $c_{k}=c_{k, d}=2^{k} k ! \prod_{j=0}^{k-1}(d+2 j)=\Delta^{k}|x|^{2 k}$ are uniformly spherically convergent in a domain $\mathcal{D} \subset \mathbb{T}^{d}$. If $F-F^{\prime}=G$, where $G \in C^{2 k+4}(\mathcal{D})$, and $\Delta^{k} G=0$ in $\mathcal{D}$, then the series $S-S^{\prime}$ is summable $(B-R, \gamma+d-1)$ to 0 uniformly on every closed domain $\mathcal{D}^{\prime}$ of $\mathcal{D}$.

We need the following generalization of Lemma 2 of Shapiro [Sh3].

Lemma 7. Let $B\left(x_{0}, h_{0}\right) \subset \mathbb{T}^{d}$ be an open ball. Suppose a multiple trigonometric series $\sum_{\xi} a_{\xi} e^{i\langle\xi, x\rangle}$ satisfies the following conditions:

1. the coefficients $\left\{a_{\xi}\right\}$ satisfy condition (2.3) and $\bar{a}_{\xi}=a_{-\xi}$ for all $\xi$;

2. $f^{*}(x)=\lim \sup _{t \rightarrow 0^{+}} \sum_{\xi} a_{\xi} e^{i\langle\xi, x\rangle-|\xi| t}$ is finite for all $x \in B\left(x_{0}, h_{0}\right)$; similarly, $f_{*}(x)$ is finite for all $x \in B\left(x_{0}, h_{0}\right)$; and

3. $f^{*}(x)=f_{*}(x)=0$ for almost all $x \in B\left(x_{0}, h_{0}\right)$.

Then, $\sum_{\xi} a_{\xi} \prod_{j=1}^{d} \xi_{j}^{t_{j}} e^{i\langle\xi, x\rangle}$ is summable $(B-R, t+d)$ to 0 for any lattice point $\left\langle t_{i}\right\rangle=\left\langle t_{1}, \cdots, t_{d}\right\rangle \in \mathbb{N}^{d}$ uniformly in any closed ball $B_{1} \subset B\left(x_{0}, h_{0}\right)$ where $t=$ $t_{1}+\cdots+t_{d} \geq 0$.

But first, we prove the following

Lemma 8. Let $B=B\left(x_{0}, h_{0}\right) \subset \mathbb{T}^{d}$ and $\sum_{\xi} a_{\xi} e^{i\langle\xi, x\rangle}$ be the multiple trigonometric series given as in Lemma 7. For $s \geq 1$, define

$$
G_{s}(x)=a_{0}|x|^{2 s} / c_{s}+\lim _{t \rightarrow 0+} \sum_{|\xi| \neq 0} \frac{(-1)^{s} a_{\xi}}{|\xi|^{2 s}} e^{i\langle\xi, x\rangle-|\xi| t} .
$$

Then $G_{s}(x)$ is spherically Abel convergent and is finite everywhere in B. Moreover, for any ball $B_{1} \subset \bar{B}_{1} \varsubsetneqq B$, there exists a sequence of functions $\left\{h_{s}(x)\right\}_{s \geq 0}$ such that $h_{s}(x)$ is harmonic in $B_{1}$ for all $s \geq 1$, and for the functions $G_{s}^{1}(x)$ given by

$$
G_{s}^{1}(x)=\sum_{i=1}^{s} \underbrace{\Phi *(\Phi * \cdots(\Phi}_{i} * h_{s-i}) \cdots)(x)
$$

where

$$
(\Phi * h)(x)=-\frac{1}{\sigma_{d}(d-2)} \int_{B_{1}} G_{B_{1}}(x, y) h(y) d y,
$$

$G_{B_{1}}(x, y)$ is Green's function associated to $B_{1}$ and $\sigma_{d}$ is the surface area of the unit ball in $\mathbb{R}^{d}, G_{s}(x)=G_{s}^{1}(x)+h_{s}(x)$ almost everywhere in $B_{1}$. 
Proof. When $s=1, G_{1}(x)=f_{2}(x)+a_{0}|x|^{2} /(2 d)$ and the lemma is true for $G_{1}^{1}(x)=$ $h_{0}(x)=0$ and some harmonic function $h_{1}(x)$ by Lemma 2.4 of [AW1. Assume the lemma holds true for $1 \leq s \leq s_{0}$. Since $G_{s_{0}}(x)$ is finite everywhere in $B$, using the mean value theorem (as shown at the beginning of Section 2 of AW1), we have that $G_{s_{0}+1}(x)$ is spherically Abel convergent and finite everywhere in $B$. By an argument similar to (2.4) of AW1, we have that $G_{s}(x)$ is in $L^{2}\left(\mathbb{T}^{d}\right)$ for $s \geq 1$.

Because $h_{i}(x)$ is harmonic in $B_{1}$ for $i=1, \cdots, s_{0}$, we have that $G_{s_{0}}^{1}(x) \in C^{\infty}\left(B_{1}\right)$ and

$$
\begin{aligned}
\Delta G_{s_{0}+1}^{1}(x) & =G_{s_{0}}^{1}(x)+h_{s_{0}}(x) \text { for } x \in B_{1} \\
& =G_{s_{0}}(x) \text { for almost all } x \in B_{1} .
\end{aligned}
$$

Apply Lemma 2.4 of [AW1] to $B, B_{1}$, and to $\sum_{\xi \neq 0} \frac{(-1)^{s_{0}} a_{\xi}}{|\xi|^{s_{0}}} e^{i\langle\xi, x\rangle}$, and use (3.4) to see that there exists a harmonic function, $h_{s_{0}+1}(x)$ such that $G_{s_{0}+1}(x)-G_{s_{0}+1}^{1}(x)=$ $h_{s_{0}+1}(x)$ almost everywhere in $B_{1}$. This completes the proof.

Proof of Lemma 7. Let $s>d / 4+1 / 2$ and choose a ball $B_{2}$ such that $B_{1} \varsubsetneqq B_{2} \subset$ $\overline{B_{2}} \varsubsetneqq B$. Then $\sum_{|\xi| \neq 0} \frac{a_{\xi}}{|\xi|^{2 s}} e^{i\langle\xi, x\rangle}$ is absolutely and uniformly convergent since by (2.3),

$$
\begin{aligned}
\sum_{|\xi| \neq 0} \frac{\left|a_{\xi}\right|}{|\xi|^{2 s}} & \leq 2^{s} \sum_{n \geq 1} 2^{-s n} \sum_{|\xi|^{2} \sim 2^{n}}\left|a_{\xi}\right| \\
& \leq 2^{s} \sum_{n \geq 1} 2^{-s n}\left(\sum_{|\xi|^{2} \sim 2^{n}}\left|a_{\xi}\right|^{2}\right)^{1 / 2}\left(\sum_{|\xi|^{2} \sim 2^{n}} 1\right)^{1 / 2} \\
& \leq C 2^{s} \sqrt{v_{d}} \sum_{n \geq 1} 2^{-(s-1 / 2-d / 4) n}<\infty .
\end{aligned}
$$

Thus, $G_{s}(x)$ is continuous in $B$. Consequently, by Lemma $8 G_{s}(x)=G_{s}^{1}(x)+h_{s}(x)$ for all $x \in B_{2}$ if $s>d / 4+1 / 2$. So $G_{s}(x) \in C^{\infty}\left(B_{2}\right)$. Apply $\Delta$ to $G_{s}(x) s$ times to obtain

$$
\Delta^{s} G_{s}(x)=0 \text { for } x \in B_{2} \text {, if } s>1 / 2+d / 4 .
$$

If $t=0$, apply part b) of Lemma 6 to $S(x)=\sum_{\xi} a_{\xi} e^{\langle\xi, x\rangle}$ and $S^{\prime}(x)=0$. Since $\left|a_{\xi}\right|=o(|\xi|)$ by (2.3), we may choose $\gamma=1$. For $k=\lceil\gamma / 2\rceil+\lceil d / 2\rceil+1>1 / 2+d / 4$, $F(x)=G_{k}(x) \in C^{\infty}\left(B_{2}\right)$. Thus, $F(x)$ is absolutely and uniformly convergent, and $\Delta^{k} F(x)=0$. By part b) of Lemma [6, we see that $\sum_{\xi} a_{\xi} e^{i\langle\xi, x\rangle}$ is uniformly summable $(B-R, d)$ to 0 in $B_{1}$.

If $t \geq 1$, apply part b) of Lemma [6] again to $S(x)=\sum_{\xi \neq 0} a_{\xi} \prod_{i=1}^{d} \xi_{i}^{t_{i}} e^{i\langle\xi, x\rangle}$ and $S^{\prime}(x)=0$. By (2.3), we have $\left|a_{\xi} \prod_{i=1}^{d} \xi_{i}^{t_{i}}\right|=O\left(|\xi|^{t+1}\right)$. Choose $\gamma=t+1$. For $k=\lceil\gamma / 2\rceil+\lceil d / 2\rceil+1>1 / 2+d / 4$,

$$
F(x)=i^{-t}\left(\frac{\partial^{t}}{\partial^{t_{1}} x_{1} \cdots \partial^{t_{d}} x_{d}} G_{k}(x)-\frac{\partial^{t}}{\partial^{t_{1}} x_{1} \cdots \partial^{t_{d}} x_{d}} a_{0}|x|^{2 k} / c_{k}\right)
$$

since

$$
\frac{\partial^{t}}{\partial^{t_{1}} x_{1} \cdots \partial^{t_{d}} x_{d}} G_{k}(x)=\frac{\partial^{t}}{\partial^{t_{1}} x_{1} \cdots \partial^{t_{d}} x_{d}} a_{0}|x|^{2 k} / c_{k}+i^{t} \sum_{|\xi| \neq 0} \frac{(-1)^{k} a_{\xi}}{|\xi|^{2 k}} \prod_{i=1}^{d} \xi_{i}^{t_{i}} e^{i\langle\xi, x\rangle} .
$$


By (3.5), when $s>1 / 2+d / 4$,

$$
\Delta^{s} \frac{\partial^{t}}{\partial^{t_{1}} x_{1} \cdots \partial^{t_{d}} x_{d}} G_{s}(x)=0 \text { for } x \in B_{2} .
$$

So $\Delta^{k} F=0$ since $t \geq 1$. Therefore, by part b) of Lemma $6 \sum_{\xi} a_{\xi} \prod_{j=1}^{d} \xi_{j}^{t_{j}} e^{i\langle\xi, x\rangle}$ is uniformly summable $(B-R, t+d)$ to 0 in $B_{1}$.

Now we give an analogue of Lemma 4 of Shapiro Sh2] concerning formal multiplication of multiple trigonometric series.

Lemma 9. Let $\sum_{\xi} a_{\xi} e^{i\langle\xi, x\rangle}$ be a multiple trigonometric series satisfying the conditions in Lemma 7 . Suppose that $\lambda(x)$ is a continuous periodic function of period $2 \pi$ in each variable and that its Fourier coefficients satisfy $\alpha_{\xi}=O\left(|\xi|^{-(8 d+4)}\right)$. Set $A_{\mu}=\sum_{\xi} a_{\xi} \alpha_{\mu-\xi}$. Then,

$$
\lim _{R \rightarrow \infty} \sum_{|\mu| \leq R}\left[A_{\mu}-\lambda\left(x_{0}\right) a_{\mu}\right] e^{i\left\langle\mu, x_{0}\right\rangle}\left(1-|\mu|^{2} / R^{2}\right)^{2 d+1}=0 .
$$

Proof. Without loss of generality, assume $x_{0}=0$. Changing $\alpha_{0}$ to be $\alpha_{0}-\lambda(0)$ if necessary, we may assume also that $\lambda(0)=0$ and

$$
\lim _{R \rightarrow \infty} \sum_{|\xi| \leq R} \alpha_{\xi}=0
$$

Thus, we need only to show that

$$
\sum_{|\mu| \leq R} A_{\mu}\left(R^{2}-|\mu|^{2}\right)^{2 d+1}=o\left(R^{4 d+2}\right) .
$$

Write

$$
\begin{aligned}
\sum_{|\mu| \leq R} A_{\mu}\left(R^{2}-|\mu|^{2}\right)^{2 d+1}= & \sum_{|\mu| \leq R} \sum_{\xi} a_{\xi} \alpha_{\mu-\xi}\left(R^{2}-|\mu|^{2}\right)^{2 d+1} \\
= & \sum_{|\mu| \leq R} \sum_{|\xi| \geq 2 R} a_{\xi} \alpha_{\mu-\xi}\left(R^{2}-|\mu|^{2}\right)^{2 d+1} \\
& +\sum_{|\mu| \leq R} \sum_{R+2 \leq|\xi|<2 R} a_{\xi} \alpha_{\mu-\xi}\left(R^{2}-|\mu|^{2}\right)^{2 d+1} \\
& +\sum_{|\mu| \leq R} \sum_{R<|\xi|<R+2} a_{\xi} \alpha_{\mu-\xi}\left(R^{2}-|\mu|^{2}\right)^{2 d+1} \\
& +\sum_{|\mu| \leq R|\xi \xi| \leq R} a_{\xi} \alpha_{\mu-\xi}\left(R^{2}-|\mu|^{2}\right)^{2 d+1} \\
= & A+B+C+D .
\end{aligned}
$$

We show that each of $A, B, C$ and $D$ is $o\left(R^{4 d+2}\right)$. This will complete the proof.

A). Since $\left|\alpha_{\xi}\right|=O\left(|\xi|^{-(8 d+4)}\right)$, when $|\xi| \geq 2 R$, we have

$$
\begin{aligned}
\sum_{|\mu| \leq R}\left|\alpha_{\mu-\xi}\right| & \leq \sum_{|\mu-\xi| \geq|\xi|-R}\left|\alpha_{\mu-\xi}\right| \\
& =O\left(\sum_{|P| \geq|\xi|-R} \frac{1}{|P|^{8 d+4}}\right) \\
& =O\left((|\xi|-R)^{-(7 d+4)}\right) .
\end{aligned}
$$


By (2.3), $\left|a_{\xi}\right|=o(|\xi|)$. Thus,

$$
\begin{aligned}
|A| & \leq \sum_{|\xi| \geq 2 R} \sum_{|\mu| \leq R}\left|a_{\xi}\right|\left|\alpha_{\mu-\xi}\right|\left(R^{2}-|\mu|^{2}\right)^{2 d+1} \\
& \leq \sum_{|\xi| \geq 2 R}\left|a_{\xi}\right| R^{4 d+2} \sum_{|\mu| \leq R}\left|\alpha_{\mu-\xi}\right| \\
& =o\left(R^{4 d+2} \sum_{|\xi| \geq 2 R}|\xi|(|\xi|-R)^{-(7 d+4)}\right) \\
& =o\left(R^{-2 d-1}\right) .
\end{aligned}
$$

So $A=o\left(R^{4 d+2}\right)$.

$B)$. Note

$$
\left(R^{2}-|\mu|^{2}\right)=R^{2}-|\xi|^{2}-|\mu-\xi|^{2}+2\langle\mu-\xi, \xi\rangle
$$

So, for any $k \geq 0$,

$$
\begin{aligned}
\left(R^{2}-|\mu|^{2}\right)^{k} & =\sum_{j=0}^{k} c_{j, k}\left(R^{2}-|\xi|^{2}\right)^{j}\left(|\xi|^{2}-|\mu|^{2}\right)^{k-j} \\
& =\sum_{j+l+t=k} c_{j, l,\left\langle t_{i}\right\rangle}\left(R^{2}-|\xi|^{2}\right)^{j}|\mu-\xi|^{2 l} \prod_{i=1}^{d}\left(\mu_{i}-\xi_{i}\right)^{t_{i}} \xi_{i}^{t_{i}} \\
& \leq\left.\left.\sum_{j+n+s=k} d_{j, n, s}\left|R^{2}-\right| \xi\right|^{2}\right|^{j}|\mu-\xi|^{2 n+s}|\xi|^{s}
\end{aligned}
$$

where for $\left\langle t_{i}\right\rangle=\left\langle t_{1}, \cdots, t_{d}\right\rangle \in \mathbb{N}^{d}, t=t_{1}+\cdots+t_{d}$ and $\mu=\left(\mu_{1}, \cdots, \mu_{d}\right)$. Thus,

$$
\begin{aligned}
|B| & \leq \sum_{R+2 \leq|\xi|<2 R}\left|a_{\xi}\right| \sum_{|\mu| \leq R}\left|\alpha_{\mu-\xi}\right|\left(R^{2}-|\mu|^{2}\right)^{2 d+1} \\
& =O\left(\left.\left.\sum_{R+2 \leq|\xi|<2 R}\left|a_{\xi}\right| \sum_{j+s=2 d+1}\left|R^{2}-\right| \xi\right|^{2}\right|^{j}|\xi|^{s} \sum_{|\mu| \leq R}\left|\alpha_{\mu-\xi}\right||\mu-\xi|^{4 d+2}\right) \\
& =o\left(R^{2 d+2} \sum_{R+2 \leq|\xi|<2 R}(|\xi|-R)^{2 d+1}(|\xi|-R)^{-(3 d+2)}\right) \\
& =o\left(R^{3 d+1}\right),
\end{aligned}
$$

since when $R+2 \leq|\xi|<2 R$,

$$
\begin{aligned}
\sum_{|\mu| \leq R}\left|\alpha_{\mu-\xi}\right||\mu-\xi|^{4 d+2} & =O\left(\sum_{|\mu-\xi| \geq|\xi|-R}\left|\alpha_{\mu-\xi}\right||\mu-\xi|^{4 d+2}\right) \\
& =O\left(|| \xi|-R|^{-(3 d+2)}\right) .
\end{aligned}
$$

So $B=o\left(R^{4 d+2}\right)$. 
C). As in B),

$$
\begin{aligned}
|C| & \leq \sum_{R<|\xi|<R+2}\left|a_{\xi}\right| \sum_{|\mu| \leq R}\left|\alpha_{\mu-\xi}\right|\left(R^{2}-|\mu|^{2}\right)^{2 d+1} \\
& =O\left(\left.\left.\sum_{R<|\xi|<R+2}\left|a_{\xi}\right| \sum_{j+s=2 d+1}\left|R^{2}-\right| \xi\right|^{2}\right|^{j}|\xi|^{s} \sum_{|\mu| \leq R}\left|\alpha_{\mu-\xi}\right||\mu-\xi|^{4 d+2}\right) \\
& =O\left(R^{2 d+1} \sum_{R-2 \leq|\xi|<R+2}\left|a_{\xi}\right|\right),
\end{aligned}
$$

since

$$
\sum_{|\mu| \leq R}\left|\alpha_{\mu-\xi}\right||\mu-\xi|^{4 d+2}=O\left(\sum_{|\xi| \neq 0}|\xi|^{-(4 d+2)}\right)<\infty
$$

and $R^{2}-|\xi|^{2}=O(R)$ if $R<|\xi|<R+2$. Next use (2.3) and the Cauchy-Schwarz inequality to establish a bound that will be used again later:

$$
\begin{aligned}
\sum_{|\xi| \leq R}\left|a_{\xi}\right| & \leq\left(\sum_{2^{n} \leq R^{2}} \sum_{|\xi|^{2} \sim 2^{n}}\left|a_{\xi}\right|^{2}\right)^{1 / 2}\left(\sum_{|\xi| \leq R} 1\right)^{1 / 2} \\
& =o\left(R^{1+d / 2}\right)
\end{aligned}
$$

We finally get $C=o\left(R^{2 d+2+d / 2}\right)=o\left(R^{4 d+2}\right)$. have

D). Since $\alpha_{\xi}=O\left(|\xi|^{-(8 d+4)}\right)$, for any $\left\langle t_{i}\right\rangle=\left\langle t_{1}, \cdots, t_{d}\right\rangle \in \mathbb{N}^{d}$, and $l \geq 0$, we

$$
\lim _{R \rightarrow \infty} \sum_{|\mu| \leq R} \alpha_{\mu-\xi}|\mu-\xi|^{2 l} \prod_{i=1}^{d}\left(\mu_{i}-\xi_{i}\right)^{t_{i}}=C_{l,\left\langle t_{i}\right\rangle}
$$

provided that $2 l+t_{1}+\cdots t_{d}=2 l+t \leq 4 d+2$, where $\mu=\left(\mu_{1}, \cdots, \mu_{d}\right)$. Therefore,

$$
\sum_{|\mu| \leq R} \alpha_{\mu-\xi}|\mu-\xi|^{2 l} \prod_{i=1}^{d}\left(\mu_{i}-\xi_{i}\right)^{t_{i}}=C_{l,\left\langle t_{i}\right\rangle}-\sum_{|\mu|>R} \alpha_{\mu-\xi}|\mu-\xi|^{2 l} \prod_{i=1}^{d}\left(\mu_{i}-\xi_{i}\right)^{t_{i}}
$$


In particular, $C_{0,\langle 0\rangle}=0$ by (3.6). So using (3.7) and (3.9), we have

$$
\begin{aligned}
D= & \sum_{j+l+t=2 d+1} C_{j, l,\left\langle t_{i}\right\rangle} C_{l,\left\langle t_{i}\right\rangle} \sum_{|\xi| \leq R} a_{\xi} \prod_{i=1}^{d} \xi_{i}^{t_{i}}\left(R^{2}-|\xi|^{2}\right)^{j} \\
& -\sum_{j+l+t=2 d+1} \sum_{|\xi| \leq R} C_{j, l,\left\langle t_{i}\right\rangle} a_{\xi} \prod_{i=1}^{d} \xi_{i}^{t_{i}}\left(R^{2}-|\xi|^{2}\right)^{j} \sum_{|\mu|>R} \alpha_{\mu-\xi}|\mu-\xi|^{2 l} \prod_{i=1}^{d}\left(\mu_{i}-\xi_{i}\right)^{t_{i}} \\
= & \sum_{j+l+t=2 d+1,} C_{j, l,\left\langle t_{i}\right\rangle} C_{l,\left\langle t_{i}\right\rangle} \sum_{|\xi| \leq R} a_{\xi} \prod_{i=1}^{d} \xi_{i}^{t_{i}}\left(R^{2}-|\xi|^{2}\right)^{j} \\
& +\sum_{j+l+t=2 d+1,} C_{j, l,\left\langle t_{i}\right\rangle} C_{l,\left\langle t_{i}\right\rangle} \sum_{|\xi| \leq R} a_{\xi} \prod_{i=1}^{d} \xi_{i}^{t_{i}}\left(R^{2}-|\xi|^{2}\right)^{j} \\
& \quad-\sum_{j+l+t=2 d+1} C_{j, l,\left\langle t_{i}\right\rangle} \sum_{|\xi| \leq R} a_{\xi} \prod_{i=1}^{d} \xi_{i}^{t_{i}}\left(R^{2}-|\xi|^{2}\right)^{j} \sum_{|\mu|>R} \alpha_{\mu-\xi}|\mu-\xi|^{2 l} \prod_{i=1}^{d}\left(\mu_{i}-\xi_{i}\right)^{t_{i}} \\
= & +F-G
\end{aligned}
$$

where $j_{0}$ is the largest integer smaller than or equal to $3 d / 2$.

We estimate $E$ first. Using (3.8), we have

$$
|E| \leq o\left(R^{2+d / 2+2 d+j_{0}}\right)=o\left(R^{4 d+2}\right) .
$$

Next, if $2 d+1 \geq j \geq j_{0}+1 \geq 3 d / 2+1 / 2$, then $j+l+t=2 d+1$ implies that $t+d \leq 3 d+1-j \leq 3 d / 2+1 / 2 \leq j$. So by Lemma 7 .

$$
\sum_{|\xi| \leq R} a_{\xi} \prod_{i=1}^{d} \xi_{i}^{t_{i}}\left(R^{2}-|\xi|^{2}\right)^{j}=o\left(R^{2 j}\right)=o\left(R^{4 d+2}\right) .
$$

This shows that $F=o\left(R^{4 d+2}\right)$. Finally, using (3.8),

$$
\begin{aligned}
|G| & \leq \sum_{j+l+t=2 d+1} \sum_{|\xi| \leq R}\left|a_{\xi}\right| R^{t+j}(R-|\xi|)^{j} \sum_{|\mu-\xi|>R-|\xi|}\left|\alpha_{\mu-\xi}\right||\mu-\xi|^{4 d+2} \\
& =o\left(R^{2+d / 2+2 d}\right) .
\end{aligned}
$$

This completes the proof.

The next lemma shows that if a multiple trigonometric series has rapidly decaying coefficients, then formal multiplication by that series preserves the Bourgain condition (2.3).

Lemma 10. If $\left\{a_{\xi}\right\}_{\xi}$ satisfies (2.3):

$$
\sum_{|\xi| \sim R}\left|a_{\xi}\right|^{2}=o\left(R^{2}\right)
$$

and if $\left\{\alpha_{\xi}\right\}_{\xi}$ satisfies

$$
\left|\alpha_{\xi}\right|=O\left(|\xi|^{-(2 d+1)}\right) \text { as }|\xi| \rightarrow \infty,
$$

then $\left\{A_{\mu}\right\}_{\mu}=\left\{\sum_{\xi} a_{\mu-\xi} \alpha_{\xi}\right\}_{\mu}$ satisfies

$$
\sum_{|\mu| \sim R}\left|A_{\mu}\right|^{2}=o\left(R^{2}\right)
$$


as $R \rightarrow \infty$.

Proof. Set $\beta=\frac{d}{2(d+1)}$. Note

$$
\begin{aligned}
\sum_{|\mu| \sim R}\left|A_{\mu}\right|^{2} & =\sum_{|\mu| \sim R}\left|\sum_{\xi} a_{\mu-\xi} \alpha_{\xi}\right|^{2} \\
& \leq 2 \sum_{|\mu| \sim R}\left|\sum_{|\xi| \leq R^{\beta}} a_{\mu-\xi} \alpha_{\xi}\right|^{2}+2 \sum_{|\mu| \sim R}\left|\sum_{|\xi| \geq R^{\beta}} a_{\mu-\xi} \alpha_{\xi}\right|^{2} \\
& =2 \mathrm{I}+2 \mathrm{II} .
\end{aligned}
$$

It follows from (2.3) that $a_{\xi}=o(|\xi|)$. So $a_{\mu-\xi}=o(|\xi|+R)$. Thus,

$$
\begin{aligned}
\mathrm{II} & =o\left(\sum_{|\mu| \sim R}\left|\sum_{|\xi| \geq R^{\beta}}(|\xi|+R)\right| \alpha_{\xi}||^{2}\right) \\
& =o\left(R^{2} \sum_{|\mu| \sim R} R^{-2(d+1) \beta}\right) \\
& =o\left(R^{2}\right)
\end{aligned}
$$

since $\sum_{|\xi| \geq R^{\beta}}\left|\alpha_{\xi}\right|=O\left(R^{-(d+1) \beta}\right)$ and $\sum_{|\xi| \geq R^{\beta}}|\xi|\left|\alpha_{\xi}\right|=O\left(R^{-d \beta}\right)$.

To show that $\mathrm{I}=o\left(R^{2}\right)$, it is enough to show that

$$
\mathrm{III}=\sum_{|\mu| \sim R}\left|\sum_{0<|\xi| \leq R^{\beta}} a_{\mu-\xi} \alpha_{\xi}\right|^{2}=o\left(R^{2}\right) .
$$

Let $2^{n-1}<R^{2 \beta} \leq 2^{n}$. By the Cauchy-Schwarz inequality, we have

$$
\begin{aligned}
\text { III } & \leq \sum_{|\mu| \sim R}\left(\sum_{k=1}^{n} \sum_{|\xi|^{2} \sim 2^{k}}\left|a_{\mu-\xi}\right|\left|\alpha_{\xi}\right|\right)^{2} \\
& \leq \sum_{|\mu| \sim R}\left(\sum_{k=1}^{n}\left(\sum_{|\xi|^{2} \sim 2^{k}}\left|a_{\mu-\xi}\right|^{2}\right)^{1 / 2}\left(\sum_{|\xi|^{2} \sim 2^{k}}\left|\alpha_{\xi}\right|^{2}\right)^{1 / 2}\right)^{2} \\
& =O\left(\sum_{|\mu| \sim R}\left(\sum_{k=1}^{n} \frac{1}{2^{3 k d / 4+k / 2}}\left(\sum_{|\xi|^{2} \sim 2^{k}}\left|a_{\mu-\xi}\right|^{2}\right)^{1 / 2}\right)^{2}\right) \\
& =O\left(\sum_{|\mu| \sim R}\left(\sum_{k=1}^{n} \frac{1}{2^{k}}\right)\left(\sum_{k=1}^{n} \frac{1}{2^{3 k d / 2}} \sum_{|\xi|^{2} \sim 2^{k}}\left|a_{\mu-\xi}\right|^{2}\right)\right) \\
& =O\left(\sum_{k=1}^{n} \frac{1}{2^{3 k d / 2}} \sum_{|\xi|^{2} \sim 2^{k}}\left(\sum_{|\mu| \sim R}\left|a_{\mu-\xi}\right|^{2}\right)\right)
\end{aligned}
$$


For $R$ large, $R^{\beta} \ll R$. So $R / 4<|\mu-\xi|<5 R / 4$, if $|\mu| \sim R,|\xi| \leq R^{\beta}$, and $R$ is large. Thus by (2.3), for $R$ large, and for each $\xi$ satisfying $|\xi| \sim 2^{k}$,

$$
\sum_{|\mu| \sim R}\left|a_{\mu-\xi}\right|^{2} \leq \sum_{R / 4<|\mu-\xi|<5 / 4 R}\left|a_{\mu-\xi}\right|^{2}=o\left(R^{2}\right) .
$$

Consequently, by 3.11) we have

$$
\begin{aligned}
\text { III } & =o\left(R^{2} \sum_{k=1}^{n} \frac{1}{2^{3 k d / 2}} \sum_{|\xi|^{2} \sim 2^{k}} 1\right) \\
& =o\left(R^{2} \sum_{k=1}^{n} 2^{-k d}\right) \\
& =o\left(R^{2}\right) .
\end{aligned}
$$

This finishes the proof.

\section{Proof of Theorem 7}

Let $H$ be an $H^{J}$ set. Suppose that

$$
\lim _{t \rightarrow 0^{+}} \sum a_{\xi} e^{i\langle\xi, x\rangle-|\xi| t}=0
$$

for $x \in \mathbb{T}^{d} \backslash H$ and that $\left\{a_{\xi}\right\}$ satisfies condition (2.2). Because of Lemma 4, we may assume that $H$ is closed. Without loss of generality, we may also assume that $\bar{a}_{\xi}=a_{-\xi}$. Let $\left\{\lambda_{k}(x)\right\}_{k \geq 1}$ be the sequence of $C^{\infty}\left(\mathbb{R}^{d}\right)$ functions given by Lemma 5 and denote $\left\{\alpha_{\xi}^{k}\right\}$ to be their Fourier coefficients. Set $A_{\mu}^{k}=\sum_{\xi} a_{\xi} \alpha_{\mu-\xi}^{k}$. Our next goal is to show that

$$
\lim _{t \rightarrow 0^{+}} \sum_{\mu} A_{\mu}^{k} e^{i\langle\mu, x\rangle-|\mu| t}=0 \text { for all } k \geq 1 \text { and all } x \in \mathbb{T}^{d} .
$$

Let $x_{0} \in \mathbb{T}^{d}$. Then either $x_{0} \in H$ or $x_{0} \in \mathbb{T}^{d} \backslash H$. Consider the case $x_{0} \in H$ first. For all $k \geq 1$, we have $x_{0} \in D_{k}^{*}$. Thus, there exists $h_{0}>0$ such that $B=B\left(x_{0}, h_{0}\right) \in D_{k}^{*}$. Consequently, $\lambda_{k}(x)=0$ for all $x \in B$. Therefore, by part a) of Lemma [6] $\sum_{\mu} A_{\mu}^{k} e^{i\langle\mu, x\rangle}$ is summable $(B-R, d)$ to 0 uniformly in $B$. Thus, (4.2) holds for $x_{0}$ and for all $k \geq 1$.

Next assume $x_{0} \notin H$. Since $H^{*}$ is closed, there exist $h_{0}>0$, such that for all $x \in B=B\left(x_{0}, h_{0}\right)$, (4.1) holds. By Lemma 9], we have $\sum_{\mu}\left[A_{\mu}^{k}-\lambda_{k}\left(x_{0}\right) a_{\mu}\right] e^{i\left\langle\mu, x_{0}\right\rangle}$ is summable $(B-R, 2 d+1)$ to 0 , and consequently, spherically Abel convergent to 0 . Since (4.1) holds for $x_{0}$, 4.2 holds for $x_{0}$.

Now apply Lemma 10, For each $k,\left\{A_{\mu}^{k}\right\}$ satisfies (2.3). Thus, (4.2) and Theorem 6 imply that

$$
A_{\mu}^{k}=0
$$

for all $k \geq 1$ and all $\mu \in \mathbb{Z}^{d}$. We show that

$$
a_{\mu}=0
$$

for all $\mu \in \mathbb{Z}^{d}$.

Fix $\mu$. Condition (2.2) implies that $a_{\xi}=o(1)$. So, for any $\varepsilon>0$, there exists $\xi_{0}>1$ such that $\left|a_{\eta}\right|<\varepsilon / C$ for all $|\eta| \geq \xi_{0} / 2$ and $|\mu-\xi| \geq|\xi| / 2$ whenever $|\xi| \geq \xi_{0}$, 
where $C$ is the constant given in Lemma 5 . Then, (4.3) and Lemma 5 imply that

$$
\left|\alpha_{0}^{k} a_{\mu}\right| \leq \varepsilon+\sum_{1 \leq|\xi| \leq \xi_{0}}\left|a_{\mu-\xi}\right|\left|\alpha_{\xi}^{k}\right| .
$$

Let $k \rightarrow \infty$. By Lemma [5] we have $\left|a_{\mu}\right| \leq \varepsilon$. This proves the theorem.

\section{Proof of Theorem 8}

Before we prove Theorem 8 we need the following lemma, which gives a local property of closed sets of Abel uniqueness.

Lemma 11. Let $\sum_{\xi} a_{\xi} e^{i\langle\xi, x\rangle}$ be a multiple trigonometric series satisfying condition (2.3). Let

$$
f(x, t)=\sum_{\xi} a_{\xi} e^{i\langle\xi, x\rangle-|\xi| t} .
$$

Let $B=B\left(x_{0}, h_{0}\right) \subset \mathbb{T}^{d}$. If $E^{*}=\bigcup_{\mu}[E+2 \pi \mu]$ is a closed set of Abel uniqueness and

$$
\lim _{t \rightarrow 0^{+}} f(x, t)=0
$$

for all $x \in B \backslash E^{*}$, then

$$
\lim _{t \rightarrow 0^{+}} f(x, t)=0
$$

for all $x \in B$.

Proof. By periodicity, we may assume that $B=B\left(0, h_{0}\right)$, that $0 \in E$, and that we need to show that $\lim _{t \rightarrow 0^{+}} f(0, t)=0$. Choose $0<h_{3}<h_{2}<h_{1}<h_{0}$. There exists a $C^{\infty}\left(\mathbb{R}^{d}\right)$ function $\lambda(x)$, of period $2 \pi$ in each variable, such that

$$
\lambda(x)= \begin{cases}1 & \text { in } B\left(0, h_{2}\right), \\ 0 & \text { in } \mathbb{T}^{d} \backslash B\left(0, h_{1}\right) .\end{cases}
$$

Denote the Fourier coefficients of $\lambda(x)$ by $\alpha_{\xi}$ and set $A_{\mu}=\sum_{\mu} a_{\mu-\xi} \alpha_{\xi}$. Since $E^{*}$ is a closed set, for any $x_{1} \in B\left(0, h_{1}\right) \backslash E^{*}$, there exists $\rho_{1}>0$ such that $B\left(x_{1}, \rho_{1}\right) \subset B\left(0, h_{0}\right) \backslash E^{*}$. So $\lim _{t \rightarrow 0^{+}} f(x, t)=0$ in $B\left(x_{1}, \rho_{1}\right)$. Thus, by Lemma 9

$$
\lim _{t \rightarrow 0^{+}} \sum_{\mu}\left[A_{\mu}-\lambda(x) a_{\mu}\right] e^{i\langle\mu, x\rangle-|\mu| t}=0
$$

for $x \in B\left(0, h_{1}\right) \backslash E^{*}$. This combining with (5.1) shows that

$$
\lim _{t \rightarrow 0^{+}} \sum_{\mu} A_{\mu} e^{i\langle\mu, x\rangle-|\mu| t}=0
$$

for $x \in B\left(0, h_{1}\right) \backslash E^{*}$. If $x \in \mathbb{T}^{d} \backslash B\left(0, h_{1}\right)$, then $\lambda(x)=0$. Hence, by part a) of Lemma 6,

$$
\lim _{t \rightarrow 0^{+}} \sum_{\mu} A_{\mu} e^{i\langle\mu, x\rangle-|\mu| t}=0
$$

for $x \in \mathbb{T}^{d} \backslash B\left(0, h_{1}\right)$. By Lemma 10 and Lemma 1 of [Be], $A_{\mu}$ satisfies condition (2.2). Thus (15.2) and (15.3) imply that $A_{\mu}=0$ for all $\mu \in \mathbb{N}^{d}$ since $E^{*}$ is an Abel set of uniqueness. However, on $B\left(0, h_{2}\right), \lambda(x)=1$. Thus by part a) of Lemma 6 ,

$$
\lim _{t \rightarrow 0^{+}} \sum_{\mu}\left[A_{\mu}-a_{\mu}\right] e^{i\langle\mu, x\rangle-|\mu| t}=0
$$


for $x \in B\left(0, h_{3}\right)$. Consequently, $\lim _{t \rightarrow 0^{+}} f(0, t)=0$.

We finally prove Theorem 8 .

Proof of Theorem 8 . Suppose that

$$
\lim _{t \rightarrow 0^{+}} \sum_{\xi} a_{\xi} e^{i\langle\xi, x\rangle-|\xi| t}=\lim _{t \rightarrow 0^{+}} f(x, t)=0
$$

for $x \in \mathbb{R}^{d} \backslash E^{*}$ and the $\left\{a_{\xi}\right\}$ satisfies condition (2.2). Without loss of generality, assume that $\bar{a}_{\xi}=a_{-\xi}$. Our goal is to show that

$$
Z=\left\{x \in \mathbb{R}^{d}: \limsup _{t \rightarrow 0^{+}}|f(x, t)|=+\infty\right\}=\emptyset .
$$

If (5.5) holds, then $f^{*}(x)$ and $f_{*}(x)$ are everywhere finite. By Theorem [5] $E^{*}$ has measure 0 since $E_{k}^{*}$ has measure 0 for each $k \geq 1$. Thus, by (5.4), $f_{*}(x)=0$ almost everywhere. So by Theorem $6, a_{\xi}=0$ for all $\xi$. This will finish the proof.

To see (5.5), assume that $Z \neq \emptyset$. Note for each $0<r<t$, there exists $r<s<t$ such that

$$
\sup _{x \in \mathbb{R}^{d}}|f(x, t)-f(x, r)| \leq \sum_{\xi}\left|a_{\xi}\right||\xi| e^{-|\xi| s}(t-r) .
$$

Because of this, each interval $[1 /(i+1), 1 / i], i \geq 1$, can be partitioned by a finite number of points $t_{i, k}$ such that

$$
\sup _{k} \sup _{\substack{x \in \mathbb{R}^{d}, t \in\left[t_{i, k+1}, t_{i, k}\right]}}\left|f(x, t)-f\left(x, t_{i, k}\right)\right| \leq 1 .
$$

Splice these partitions together to produce a decreasing to 0 sequence $\left\{t_{k}\right\}_{k \geq 1}$ with $t_{1}=1$ such that

$$
\sup _{k \geq 1} \sup _{\substack{x \in \mathbb{R}^{d} \\ t_{k+1} \leq t \leq t_{k}}}\left|f(x, t)-f\left(x, t_{k}\right)\right| \leq 1 .
$$

Then

$$
Z=\bigcap_{j=1}^{\infty} \bigcup_{k=1}^{\infty}\left\{x:\left|f\left(x, t_{k}\right)\right|>j\right\}
$$

So $Z$ is a $G_{\delta}$ set.

Condition (5.4) implies that $Z \subset E^{*}$. So $Z=\bigcup_{k}\left(Z \cap E_{k}^{*}\right)$. Apply the Baire Category Theorem relative to the space $Z$ to obtain $k_{0}>0$ and a ball $B\left(x_{0}, h_{0}\right)$ such that $B\left(x_{0}, h_{0}\right) \cap Z \subset E_{k_{0}}^{*}$ and $B\left(x_{0}, h_{0}\right) \cap Z \neq \emptyset$. Thus,

$$
\limsup _{t \rightarrow 0^{+}}|f(x, t)|<\infty \text { for all } x \in B\left(x_{0}, h_{0}\right) \backslash E_{k_{0}}^{*} \text {. }
$$

Since $E_{k_{0}}^{*}$ is closed, for any $x_{1} \in B\left(x_{0}, h_{0}\right) \backslash E_{k_{0}}^{*}$, there exists $\rho_{1}>0$ such that $B\left(x_{1}, \rho_{1}\right) \subset B\left(x_{0}, h_{0}\right) \backslash E_{k_{0}}^{*}$. Thus (5.6) implies that

$$
\limsup _{t \rightarrow 0^{+}}|f(x, t)|<\infty \text { for all } B\left(x_{1}, \rho_{1}\right) .
$$

Since $E^{*}$ has measure 0, (5.4) implies that $f_{*}(x)=0$ almost everywhere in $B\left(x_{1}, \rho_{1}\right)$. Thus, Lemma 2.5 of AW1 implies that

$$
\lim _{t \rightarrow 0^{+}} f(x, t)=0 \text { for all } x \in B\left(x_{1}, \rho_{1}\right) .
$$


Thus,

$$
\lim _{t \rightarrow 0^{+}} f(x, t)=0 \text { for all } x \in B\left(x_{0}, h_{0}\right) \backslash E_{k_{0}}^{*} .
$$

Now apply Lemma 11 We have $\lim _{t \rightarrow 0^{+}} f(x, t)=0$ for all $x \in B\left(x_{0}, h_{0}\right)$. Thus, $B\left(x_{0}, h_{0}\right) \cap Z=\emptyset$, which contradicts $B\left(x_{0}, h_{0}\right) \cap Z \neq \emptyset$. This completes the proof.

\section{REFERENCES}

[AKR] J. M. Ash, E. Rieders, and R. P. Kaufman, The Cantor-Lebesgue property, Israel J. Math. 84 (1993), 179-191. MR 94m:42007

[AW] J. M. Ash and G. Wang, A survey of uniqueness questions in multiple trigonometric series, A Conference in Harmonic Analysis and Nonlinear Differential Equations in Honor of Victor L. Shapiro, Contemporary Mathematics, 208 (1997), 35-71. MR 99f:42019

[AW1] J. M. Ash and G. Wang, Some spherical uniqueness theorems for multiple trigonometric series, Annals of Math., 151 (2000), 1-33. MR 2001a:42009

[AW2] J. M. Ash and G. Wang, Sets of uniqueness of the power of the continuum, Proc. of the Georgian Academy of Sciences, 122 (2000), 15-19. MR 2001k:42010

[Be] L. D. Berkovitz, Circular summation and localization of double trigonometric series, Trans. Amer. Math. Soc. 70 (1951), 323-344. MR 12:697b

[B] J. Bourgain, Spherical summation and uniqueness of multiple trigonometric series, Internat. Math. Res. Notices (1996), 93-107. MR 97b:42022

[Co] B. Connes, Sur les coefficients des séries trigonométriques convergentes sphériquement, C. R. Acad. Sci. Paris Sér. A 283 (1976), 159-161. MR 54:10975

[C] R. Cooke, A Cantor-Lebesgue theorem in two dimensions, Proc. Amer. Math. Soc. 30 (1971), 547-550. MR 43:7847

[KL] A. S. Kechris and A. Louveau, Descriptive set theory and the structure of sets of uniqueness, London Mathematical Society Lecture Note Series, 128, Cambridge University Press, Cambridge-New York, 1987. MR 90a:42008

[KN] L. Kuipers and H. Niederreiter, Uniform Distribution of Sequences, Wiley, New York, 1974. MR 54:7415

[PS] I. I. Pyatetski-Shapiro, On the problem of uniqueness of expansion of a function in a trigonometric series (in Russian), Učenye Zapiski Moskovskogo Gosudarstvennogo Universiteta, 155, Matematika 5 (1952), 54-72.

[PS1] I. I. Pyatetski-Shapiro, Supplement to the work 'On the problem, etc.' (in Russian), Učenye Zapiski Moskovskogo Gosudarstvennogo Universiteta, 165, Matematika 7(1954), 78-97.

[Sh] V. L. Shapiro, Uniqueness of multiple trigonometric series, Ann. Math. 66 (1957), 467480. MR 19:854d

[Sh1] V. L. Shapiro, Fourier series in several variables, Bull. Amer. Math. Soc. 70 (1964), 48-93. MR 28:1448

[Sh2] V. L. Shapiro, Removable sets for pointwise solutions of the generalized Cauchy-Riemann equations, Ann. Math. 92 (1970), 82-101. MR 55:10819

[Sh3] V. L. Shapiro, Sets of uniqueness on the 2- torus, Trans. Amer. Math. Soc. 165 (1972), 127-147. MR 46:7798

[Z] A. Zygmund, A Cantor-Lebesgue theorem for double trigonometric series, Studia Math. 43 (1972), 173-178. MR 47:711

[Z1] A. Zygmund, Trigonometric series, Vols. I, II, Cambridge University Press, CambridgeNew York-Melbourne, 1977. MR 58:29731

Mathematics Department, DePaul University, Chicago, Illinois 60614

E-mail address: mash@math.depaul.edu

Mathematics Department, DePaul University, Chicago, Illinois 60614

E-mail address: gwang@math.depaul.edu 